\title{
Sequential phonological effects in recitation times
}

\author{
CARL P. BROWMAN and DANIEL C. O'CONNELL \\ Saint Louis University, Saint Louis, Missouri 63103
}

\begin{abstract}
Sixty subjects were presented ordered letter pairs separated by two, four, or six positions and instructed to recite the sequence inclusive of the two letters presented. Half of the subjects recited overtly and the remaining half recited covertly. Earlier studies have suggested that differential recitation times are due to differences in difficulty of "entry" into the alphabet, which relates to alphabetic position. However, the results of the present investigation did not support this hypothesis. The distribution of recitation times was generally more similar in shape when comparisons of a single sequence length were made across groups (overt vs. covert) than of unequal sequence lengths within a group. Data are discussed in terms of phonological transition.
\end{abstract}

Recent studies (Lovelace \& Spence, 1972; Lovelace, Powell, \& Brooks, 1973) have suggested that differential reaction times (RTs) in alphabetic recitation are related to the degree of difficulty in "entering" the alphabet. Lovelace and Spence (1972) reported generally increased RTs near the end of the alphabet to recite the letter following the one presented. In a later study, Lovelace et al. (1973) reported the same systematic relationship between letter positions and the time required to recite a sequence of letters: RTs were generally longer for sequences of letters near the end of the alphabet than for those near the beginning of the alphabet. Lovelace et al. (1973) concluded that difficulty in entering the alphabet increases toward the end of the alphabet and difficulty in moving from letter to letter for any sequence is constant once the recitation has started. Such an explanation assumes that the basic shape of the function relating RTs to the ordered sequences of a given length is constant. Adding letters to the sequences recited would merely elevate the entire function without significantly changing its shape.

Another explanation is equally tenable. Differential RTs may reflect differences in the phonetic and articulatory characteristics of each sequence. This hypothesis would predict that the distribution of RTs for sequences of different letter lengths would not necessarily be identical. However, the shape of the distribution of RTs for sequences of the same letter length between overt and covert recitation groups would be expected to display the highest degree of similarity. That is, the same phonetic transitions are involved in a particular sequence whether recited overtly or covertly.

The present study sought to test both of these hypotheses. Subjects were instructed to recite, either overtly or covertly, a sequence of letters separated by two, four, or six alphabetic positions when visually presented with the initial and terminal letters of the sequence.

Address requests for reprints to Carl P. Browman, Department of Psychology, Saint Louis University, 221 North Grand Boulevard, Saint Louis, Missouri 63103.

\section{METHOD}

Subjects were 60 undergraduates enrolled in introductory psychology courses at Saint Louis University. All subjects were right-handed, had normal or corrected vision, and spoke English as their native language.

The subjects were tested individually in a test chamber, with the experimenter and all projection and timing apparatus in an adjacent room. They were seated approximately $65 \mathrm{~cm}$ from a translucent screen and instructed to keep their left and right hands in designated areas except when pushing the response key with their right hand. Ordered pairs of letters $3.4 \mathrm{~cm}$ in height were presented on the screen. Letter pairs had separations of two, four, or six positions (one, three, or five intervening letters, respectively). Subjects recited the sequence of letters inclusive of the two letters presented, and then depressed a response key. This would automatically advance the projector to an opaque slide. There was a $3.3 \mathrm{sec}$ interval between stimulus slides, and $.9 \mathrm{sec}$ preceding every stimulus a buzzer sounded briefly. Between each of four trials of 43 stimulus slides, there was a $60 \mathrm{sec}$ rest interval. A light mounted to the right of the screen was turned on during the first and last $5 \mathrm{sec}$ of the rest period. A microphone was mounted in front of the subject so that the experimenter could monitor responses, but there was no other communication between the subject and experimenter during the session. The assignment of subjects to conditions was random, so that half recited overtly and half recited covertly.

The letter pairs each subject received, the restrictions on the randomization of the letter pairs presented, and the counterbalancing of slide trays were identical to those reported by Lovelace et al. (1973).

The slides were made from transparencies of uppercase letters of Letraset 67-18-CLN and presented as black letters on a white background by a Kodak Carousel projector. A Hunter Klockounter, which provided a visual display of the subject's RT to the nearest millisecond, was started by a photocell and stopped when the response key was depressed.

\section{RESULTS}

Subjects responded to 43 different letter pairs one time each during four trials. A median RT was calculated for each subject for each letter pair. The means of the median RTs for overt and covert recitation at the three letter separations are presented in Figure 1. The analysis was primarily concerned with the relationship in shape of these functions relating RT to ordered 


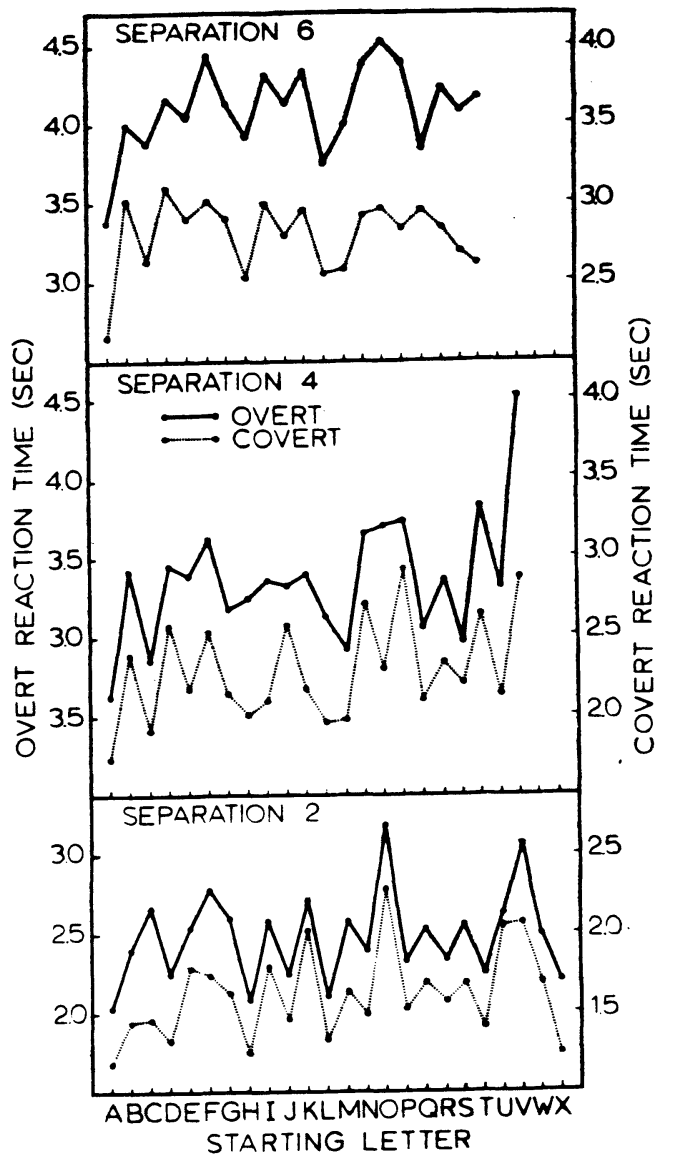

Figure 1. Means of the median reaction times (RTs) for overt and covert recitation at each letter separation. Note that RTs for overt recitation are listed on the left ordinate and RTs for covert recitation are listed on the right ordinate.

letter sequences. Product-moment correlation coefficients were calculated to determine the relative similarities in the shape of the distributions (Cattell, Couler, \& Tsujioka, 1966) at each separation and type of recitation. The correlations across letters $A$ through $\mathrm{T}, \mathrm{df}=$ 18 , relating the degree of similarity are presented in Table 1 for all possible pairs of functions. In general, same-length sequences between overt and covert recitation resulted in the highest relations in shape. The correlations in Table 1 were limited to letters A through $\mathrm{T}$ for the purpose of comparing all functions. Further calculations to include all sequences between overt and covert recitation at Separation $2, \mathrm{r}(22)=.900, \mathrm{p}<.001$, and Separation $4, \mathrm{r}(20)=.837, \mathrm{p}<.001$, indicated that these functions remained highly similar.

In overt recitation, subjects made errors in recitation on $2.79 \%$ of all possible responses. These errors decreased nearly linearly, with $38.90 \%$ occurring on the first trial and $15.98 \%$ occurring on the last trial. Less than $1 \%$ of the data was lost due to equipment failures or experimenter errors.
Table 1

Degree of Similarity between Functions

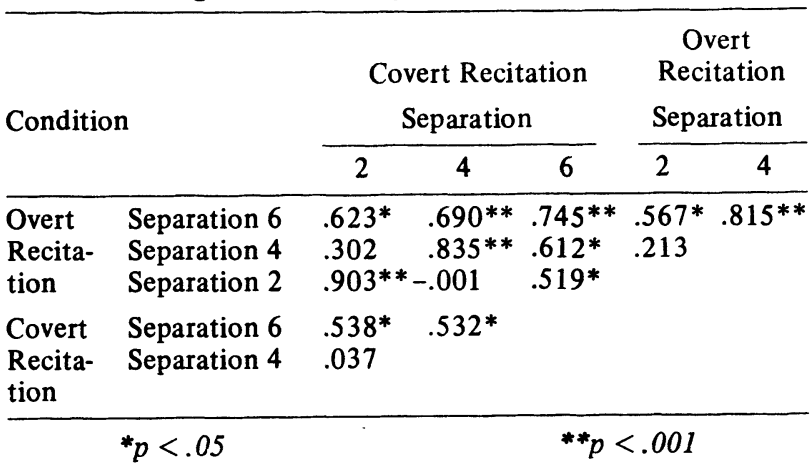

\section{DISCUSSION}

The RTs were generally slower than previously reported (Lovelace et al., 1973; Olshavsky \& Gregg, 1970). This could be the result of instructional differences. Subjects in the present study were instructed to keep their right hand in position $(3.2 \mathrm{~cm}$ in front of the response key) until after they had recited the final letter in a sequence.

The purpose of this study was to determine whether adding letters to sequences recited would uniformly or differentially elevate the functions relating RTs to order sequence position. The functions generally displayed the highest degree of similarity in overall shape when comparisons were made of the same sequence lengths between overt and covert recitation. The only exception to this was at Separation 6. The distribution for overt recitation of sequences this length was more similar in shape to the function for overt recitation of sequences at Separation 4 than to its respective covert function.

The alphabetic position effect hypothesis (Lovelace et al., 1973) would predict all the correlations in Table 1 to be approximately the same. Response latency or entry difficulty is the only determinant of RT variability under this hypothesis. Once the alphabet is entered, the recitation of all letters is constant. Hence, adding letters to the recitation would uniformly elevate the function, since it would be as difficult to enter the alphabet at $Q$ to recite $Q$ to $S$ as to recite $Q$ to $W$. The results from this study do not confirm the position effect hypothesis. It is clear that adding letters to be recited does not simply elevate the entire distribution, but also changes its shape.

The data generally support the phonological transition hypothesis. This hypothesis predicts that the differential recitation times are a function of the degree of difficulty in pronouncing each phoneme, the number of phonemes involved per letter, and the degree of difficulty in moving from one phoneme to another during recitation. The results suggest that the relative difficulty of a particular phonetic component is averaged either in or out when fewer or more letters are recited. That is, each sequence of letters is unique in terms of the phonetic and articulatory characteristics involved. Increased RTs near the end of the alphabet may reflect an increased difficulty in the phonetics and articulation of these sequences.

While the phonological transition hypothesis as an explanation has tentative merit, the design of the present investigation appears to be too insensitive to the phonetic characteristics of each sequence to adequately test it. Further research is indicated by the results obtained in this study.

\section{REFERENCES}

Cattell, R. B., Coulter, M. A., \& Tsujioka, B. The taxonometric recognition of types and functional emer- 
gents. In R. B. Cattell (Ed.), Handbook of multivariate experimental psychology. Chicago: Rand McNally, 1966.

Lovelace, E. A., Powell, C. M., \& Brooks, R. J. Alphabetic position effects in covert and overt alphabetic recitation times. Journal of Experimental Psychology, 1973, 99, 405-408.

Lovelace, E. A., \& Spence, W. A. Reaction times for naming successive letters of the alphabet. Journal of Experimental Psychology, 1972, 94, 231-233.

Olshavsky, R. W., \& GREGG, L. W. Information processing rates and task complexity. Journal of Experimental Psychology, 1970, 83, 131-135.

(Received for publication March 26, 1976.) 